\title{
Design and experiment of double planet carrier planetary gear flower transplanting mechanism
}

\author{
Xiong Zhao ${ }^{1,2}$, Hongwei Liao ${ }^{1}$, Xingxiao Ma ${ }^{1}$, Li Dai ${ }^{2}$, Gaohong $\mathrm{Yu}^{1}$, Jianneng Chen ${ }^{1 *}$ \\ (1. Faculty of Mechanical Engineering and Automation, Zhejiang Sci-Tech University, Hangzhou 310018, China; \\ 2. Key Laboratory of Zhejiang Transplanting Equipment Technology, Hangzhou 310018, China)
}

\begin{abstract}
At present, there is a lack of miniaturized and highly reliable plug seedling transplanting mechanism in flowerpots planting operation, in order to meet the needs of large displacement and high vertical uprightness for flower transplanting, the paper combined the transmission characteristics of the non-circular gear planetary gear train with the swing flexibility of the cam gear and proposed a double planet carrier planetary gear transplanting mechanism. The linkage of the mechanism performs variable speed rotation relative to the first planet carrier, the linkage serves as the second planet carrier, and the transplanting arm performs variable speed swing relative to the linkage. A mathematical model of a single planetary carrier mechanism was first established using the method of open linkage group solution domain synthesis, then established the kinematic equations of the second planet carrier and the transplanting arm. The two parts are combined to form the mathematical solution model of the proposed mechanism. The initial trajectory was planned according to the trajectory requirements of seedlings planting operation, the non-circular gear pitch curve in the first planet carrier was obtained and the length of the first planet carrier is $120 \mathrm{~mm}$. Then using the key points' angular deviation between the initial trajectory and the improved trajectory to obtain the cam parameters which driving the transplant arm, consequently determined the length of the second planet carrier is $69.25 \mathrm{~mm}$ and the length of the transplant arm is $112.4 \mathrm{~mm}$. Finally, the prototype of the mechanism was manufactured, and the test verified the correctness of the design method of the double planet carrier planetary gear flower potting transplanting mechanism. The transplanting success rate of this mechanism reached $94.43 \%$, and the plug seedlings planted in flowerpots had high uprightness. This research can provide a reference for the automatic development of research on flower transplanting machines.
\end{abstract}

Keywords: flower transplanting, double planet carrier planetary gear mechanism, non-circular, plug seedling DOI: $10.25165 /$ j.ijabe.20211402.5878

Citation: Zhao X, Liao H W, Ma X X, Dai L, Yu G H, Chen J N. Design and experiment of double planet carrier planetary gear flower transplanting mechanism. Int J Agric \& Biol Eng, 2021; 14(2): 55-61.

\section{Introduction}

With the increasing demand of society for flowers and the continuous development of the flower market, flower production is a labor-intensive industry, and the demand of automatic equipment for potted flowers is also growing ${ }^{[1]}$. The important technology of automatic flower transplanting is to design a mechanism to realize the picking of the flower seedlings and planting them into flowerpots. The mechanism that can achieve these functions is mainly divided into two types: multi-free mechanical transplanting mechanism and single-degree-of-freedom mechanical transplanting mechanism.

Feng et al. ${ }^{[2]}$ developed a flower potting mechanism based on a three-degree-of-freedom mobile platform. Hao et al. ${ }^{[3]}$ designed a

\section{Received date: $2020-04-28$ Accepted date: 2020-09-28}

Biographies: Xiong Zhao, Associate Professor, research interest: mechanism design, non-circular gear transmissions, and agricultural machinery, Email: zhaoxiong@zstu.edu.cn; Hongwei Liao, Master candidate, research interest: agricultural machinery, Email: 1153565139@qq.com; Xingxiao Ma, MS, research interest: agricultural machinery, Email: 2416771239@qq.com; Li Dai, Associate Professor, research interest: agricultural machinery, Email: dl@ zstu.edu.cn; Gaohong Yu, Professor, research interest: agricultural machinery, Email:yugh@zstu.edu.cn.

*Corresponding author: Jianneng Chen, Professor, research interest: machine design, non-circular gear transmissions and control, and agricultural machinery. Faculty of Mechanical Engineering and Automation, Zhejiang Sci-Tech University, Hangzhou 310018, China. Tel: +86-13065701536, Email: jiannengchen@zstu.edu.cn. flower potting mechanism that used a PLC to drive steel needles, Zhao et al. ${ }^{[4]}$ designed a two-degree-of-freedom hybrid-driven flower potting mechanism. Ting et al. ${ }^{[5]}$ took the adept one SCARA robot as the ontology and designed a transplanting robot with four degrees of freedom. Although these transplanting mechanisms can meet the flower plug seedlings planting function, they have not been widely used for efficiency or cost reasons.

In the research of mechanical transplanting mechanism, Dang et al. ${ }^{[6]}$ designed a single-degree-of-freedom four-barrel pick-and-plant robot arm, this mechanism combined four-link and cams, and used cams to control the clamps for taking and planting seedlings. Choi et al. ${ }^{[7]}$ studied the transplanting mechanism combined with slip-wheel system. Xin et al. ${ }^{[8]}$ designed a rice bowl seedling transplanting mechanism using a double-crank five-bar mechanism. Such mechanisms are not efficient due to transmission limitations. Zhou et al. ${ }^{[9]}$, Ye et al. ${ }^{[10]}$, Yu et al. ${ }^{[11,12]}$, and Sun et al. ${ }^{[13]}$ achieved the "eagle-mouth" transplanting trajectory design with the single planetary carrier mechanism. Zhou et al. ${ }^{[14]}$ achieved the "8" transplanting trajectory with a special non-circular planetary gear train. In order to get the ideal posture goal of the executive member of this mechanism, Zhao et al. ${ }^{[15]}$ regarded this type of mechanism as a combination of open chain rod and non-circular gear. They applied the method of open linkage group solution domain synthesis into the design of the single planetary carrier mechanism.

Although the structure of the single planet carrier planetary gear train is simple, it is not easy to achieve large displacement 
trajectory requirements. Zhao et al. ${ }^{[16]}$ designed an asymmetric high-order non-circular gear planetary gear spring onion seedling mechanism, it proved that using a second-order non-circular gear could achieve a large amount of displacement. Zhang et al. ${ }^{[17]}$ designed a single planetary carrier tomatoes seedling transplanting mechanism which combined a non-circular gear planetary gear train with a transplanting arm equipped with a seedling extraction device. It could increase the amount of mechanism displacement. Sun et al. ${ }^{[18]}$ designed a gear-linkage double planetary gear mechanism for vegetable seedling transplanting, which showed that the double planet carrier planetary gear mechanism had advantages in transplanting displacement of the mechanism.

In order to achieve the goal of picking seedlings and planting them, it is necessary to implement a large transplanting displacement of the transplanting arm. This study conducted research on a double planet carrier planetary gear flower transplanting mechanism. A set of cam pairs were added to the linkage of single planetary carrier mechanism, and the cam gear was used to control the swing of the transplanting arm ${ }^{[19,20]}$. The mechanism could ensure the uprightness of the seedlings at the planting point and large transplanting displacement. After optimization, the ideal parameters of the mechanism were obtained. A double planet carrier planetary gear flower transplanting mechanism was designed for the flowerpot planting operation. The working performance of the mechanism was verified by the flower transplanting experiment, which proved the superiority of the double planet carrier planetary gear flower potting transplanting mechanism.

\section{Structure principle of the double planet carrier planetary gear mechanism}

The single planetary carrier mechanism has the advantages of smooth transmission, non-equal speed transmission, high accuracy, and no accumulated error. This type of mechanism is composed of two parts. One part is an open chain two linkage group which is composed of a crank and a linkage. Another one is a non-circular gear train, and it combines with the previous part of the crank. Therefore, the overall freedom degree of the single planetary carrier mechanism is 1 . The double planet carrier planetary gear mechanism proposed in the study has three linkages: the first planetary carrier, the second planetary carrier, and the transplanting arm are shown in Figure 1.
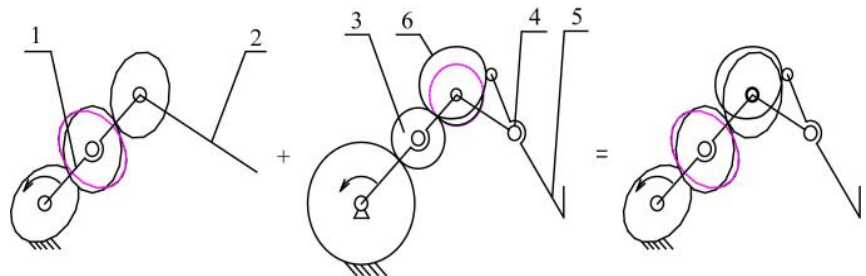

1. First planet carrier 2. Second planet carrier 3. Circle gear set 4. Third rotary joint 5 . Transplanting arm 6. Planetary gear

Figure 1 Schematic diagram of the double planet carrier planetary gear mechanism

The driving form of the second planet carrier is the same as that of the single planetary carrier mechanism, which is driven by a non-circular gear train. Therefore, the second planetary carrier will also rotate around the first planetary carrier.

Compared with the single planetary carrier mechanism, double planet carrier planetary gear mechanism adds a rotating pair at the terminal of the second planetary carrier, and this rotating pair that named the third rotary joint in this study controls the motion of the transplant arm.

In a motion cycle, the third rotating pair rotates relative to the second planetary carrier for driving the transplanting arm. The third rotating pair is driven by a set of circle gears located on the first planet carrier, and the transmission ratio difference between the circle gears and the non-circular gear train is 1 , this character makes the cam 2 turns around relative to the second planetary carrier in a cycle. The power of the third rotating pair still comes from the first planetary carrier, the overall degree of freedom of the double planet carrier planetary gear mechanism is still 1 .

Based on the kinematic requirement of large displacement of the mechanism, the double planet carrier planetary gear transplanting mechanism proposed in this study was composed of the second-order non-circular gear train and cam gear.

\section{Establishment of a mathematical model for the double planet carrier planetary gear mechanism}

According to the structure principle of the mechanism, the parameter solving model of the mechanism is established in two parts.

\subsection{Parameter solution model of the open chain two linkage group}

The calculation of the first planet carrier was solved by the method of solving the linkage groups. The single planetary carrier mechanism was divided into two parts: the linkage group and the planetary gear train. Figure 2 is a single planet carrier linkage group.

The exact attitude angle of the given three points is $P_{i}\left(x_{i}, y_{i}\right)$ $(i=1,2,3)$, the corresponding azimuth angles are $\theta_{1}, \theta_{2}, \theta_{3}$ respectively. When the fixed hinge point $O$ is $\left(x_{0}, y_{0}\right)$, combined with the displacement transformation matrix, the length of the planet carrier is constant, and Equation (1) can be obtained.

$$
\left\{\begin{array}{l}
G_{20} x_{c 1}+K_{20} y_{c 1}+N_{20}=0 \\
G_{30} x_{c 1}+K_{30} y_{c 1}+N_{30}=0
\end{array}\right.
$$

$D_{11 i}=\cos \theta_{1 i} \quad, \quad D_{12 i}=-\sin \theta_{1 i} \quad, \quad D_{13 i}=x_{i}-x_{1} \cos \theta_{1 i}+y_{1} \sin \theta_{1 i}$, $D_{21 i}=\sin \theta_{1 i} \quad, \quad D_{22 i}=\cos \theta_{1 i} \quad, \quad D_{23 i}=y_{i}-x_{1} \sin \theta_{1 i}-y_{1} \cos \theta_{1 i}$ ， $D_{31 i}=0, D_{32 i}=0, D_{33 i}=1, A_{i 1}=1-D_{11 i}, A_{i 2}=D_{12 i}, A_{i 3}=-D_{13 i}$.

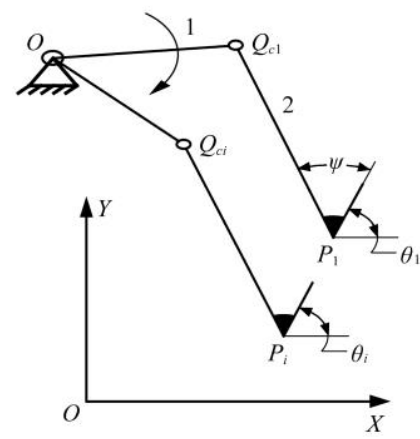

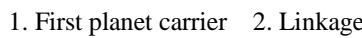

Figure 2 Linkages analysis of the single planetary carrier mechanism

$A_{i 4}=-D_{23 i}, \quad A_{i 5}=D_{11 i} D_{13 i}+D_{21 i} D_{23 i}, \quad A_{i 6}=D_{12 i} D_{13 i}+D_{22 i} D_{23 i}$, $A_{i 7}=\frac{D_{13 i}^{2}+D_{23 i}^{2}}{2}$,

$G_{20}=A_{21} x_{0}+A_{22} y_{0}+A_{25}, \quad K_{20}=A_{21} y_{0}-A_{22} x_{0}+A_{26}$

$N_{20}=A_{23} x_{0}-A_{24} y_{0}+A_{27}, \quad G_{30}=A_{31} x_{0}+A_{32} y_{0}+A_{35}$

$K_{30}=A_{31} y_{0}-A_{32} x_{0}+A_{36}, \quad N_{30}=A_{33} x_{0}+A_{34} y_{0}+A_{37}$

When the length of the first planet carrier is $r$, by Equation (2), a solution curve for the fixed hinge point $O\left(x_{0}, y_{0}\right)$ can be obtained. 


$$
\begin{aligned}
& {\left[N_{20} K_{30}-N_{30} K_{20}-x_{0}\left(G_{30} K_{20}-G_{20} K_{30}\right)\right]^{2}+} \\
& {\left[N_{20} G_{30}-N_{30} G_{20}-y_{0}\left(G_{20} K_{30}-G_{30} K_{20}\right)\right]^{2}} \\
& \quad=r^{2}\left(G_{20} K_{30}-G_{30} K_{20}\right)^{2}
\end{aligned}
$$

According to the proper position of the hinge, $x_{0}$ is limited to a range, and the value is taken at an appropriate step size. The corresponding $y_{0}$ value is obtained by Equation (2), and the solution curve of $\left(x_{0}, y_{0}\right)$ is obtained. Substituting the coordinates of the fixed hinge point $O$ into Equation (1) can obtain the solution curve of the point $Q_{c 1}\left(x_{c 1}, y_{c 1}\right)$.

Limiting the length $r(\mathrm{~mm})$ of the planetary carrier to a given range, and specifying a step value, then sets of solution curves can be obtained. For any a set of solutions associated with the first planet carrier length $r$, the parameters of the mechanism can be calculated from Equation (3), those are the linkage length $l(\mathrm{~mm})$, the initial position angles $\varphi_{11}\left({ }^{\circ}\right)$ and $\varphi_{21}\left({ }^{\circ}\right)$, and the angle $\psi\left({ }^{\circ}\right)$ between the linkage and the mark line.

$$
\left\{\begin{array}{l}
l=\sqrt{\left(x_{c 1}-x_{1}\right)^{2}+\left(y_{c 1}-y_{1}\right)^{2}} \\
\varphi_{11}=\arctan \left(\frac{y_{c 1}-y_{0}}{x_{c 1}-x_{0}}\right) \\
\varphi_{21}=\arctan \left(\frac{y_{c 1}-y_{1}}{x_{c 1}-x_{1}}\right) \\
\psi=180-\varphi_{21}-\theta_{1}
\end{array}\right.
$$

Under the condition of definite $l$, the angles at the other two precise posture points are obtained, therefore the first planetary carrier angle $\varphi_{1}\left({ }^{\circ}\right)$ and the linkage angle $\varphi_{2}\left({ }^{\circ}\right)$ in the open chain two linkage group at three precise posture points $P_{i}$ are clear. The method of cubic spline fitting is used to determine the angle curve $\varphi_{1}^{\prime}\left(^{\circ}\right)$ and $\varphi_{2}^{\prime}\left(^{\circ}\right)$ in one cycle, and then the relative rotation angle of the linkage relative to the first planetary carrier is equal to $\varphi_{2}^{\prime}$ minus $\varphi_{1}^{\prime}$, its first derivative is the total transmission ratio $i$ of the non-circular gear train in the first planet carrier.

After the transmission ratio curve is reconstructed and distributed, the relationship curve between the transmission ratio of any pair of non-circular gear in the non-circular gear train and the rotation angle of the driven gear can be obtained. The rest design process is the same as the reverse design of the non-circular planetary gear train, which has been detailed in reference [15].

\subsection{Solution of the second planet carrier and transplanting} arm

Figure 3 is a simplified diagram of a double planet carrier linkage group. The constants $r, l$, and variables $\varphi_{1}$ and $\varphi_{2}$ can be obtained from the mathematical model of the single planet carrier linkage group.

The design of the second planet carrier of double planet carrier planetary gear mechanism is shown in Figure 4. The power of the transplanting arm cam comes from the rotation of the planet carrier. The swing rod is fixedly connected with the large gear, and the large gear drives the small gear, the small gear is fixedly connected with the transplanting arm. This structure is equivalent to an angle magnification mechanism, the deflection angle of the transplanting arm is enlarged by a factor of $k$.

The cam drives for controlling the grafted arm can be divided into four sections: lifting section I, far and stop section II, return section III, and proximal stop section IV, as shown in Figure 5.

A serial of angle control points $P_{t}$ are selected on the trajectory of the open chain two linkage group, and the expectant angle displacement $\varepsilon_{t}\left({ }^{\circ}\right)$ of the swing rod is determined according to the angle of the mark line $\theta$. The rotation angle of the cam is set as $\delta$, the scope of $\delta$ is 0 to $2 \pi$. The extracted deflection angle $\varepsilon_{t}$ of the cam swing bar is placed in the corresponding angle deflection segment in order. The rotation angle of the cam and the swing angle of the cam and the swing angle of the swing rod are fitted respectively, and then the four-segment functions are spliced, a complete swing rod motion curve is obtained $\Delta \varepsilon=G(\delta)$.

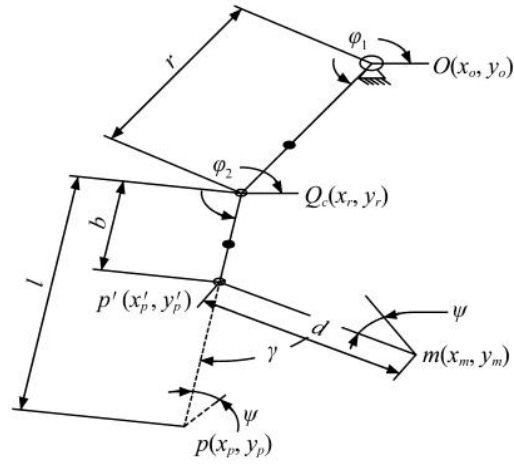

Note: The fixed hinge point $O$ is the center of revolution of the first planet carrier; the point $Q c$ is the hinge joint of the first planet carrier and the second planet carrier; $P$ is the terminal of the second planet carrier in the open chain two linkage group; $P^{\prime}$ is the actual end of the second planet carrier; $m$ is the terminal of the transplanting arm; $b$ is the actual length of the second planet carrier $(\mathrm{mm})$; $d$ is the length of the transplanting arm $(\mathrm{mm}) ; \gamma\left({ }^{\circ}\right)$ is the angle displacement of the transplanting arm.

Figure 3 Diagram of a double planet carrier linkage group

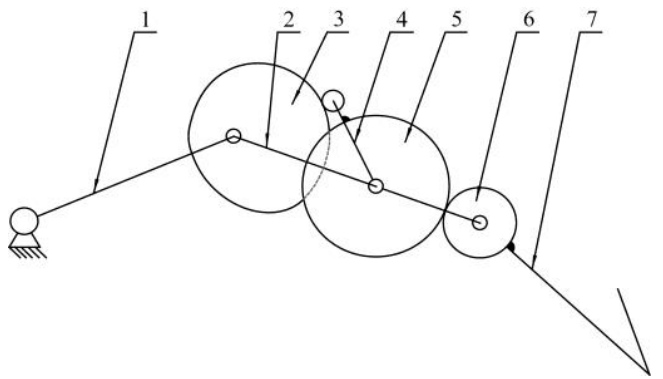

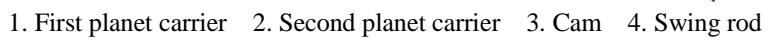
5. Large gear 6. Small gear 7. Transplanting arm

Figure 4 Driving form of the transplanting arm

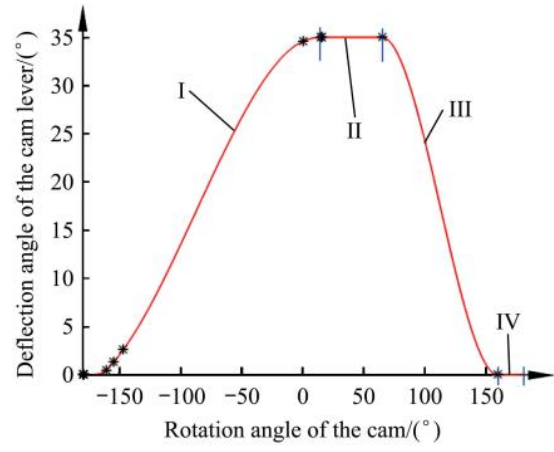

I. Lift section II. Distal stop section III. Return section IV. Proximity stop section

Figure 5 Design analysis diagram of cam

Given a base circle radius $r_{0}(\mathrm{~mm})$, a cam center distance $a_{t}$ $(\mathrm{mm})$, a roller radius $r_{r}(\mathrm{~mm})$, and a swing rod length $l_{t}(\mathrm{~mm})$. The minimum angle $\varepsilon_{\min }$ of the swing rod can be obtained from Equation (4).

$$
\varepsilon=\arccos \left(\frac{a_{t}^{2}+l_{t}^{2}-r_{0}^{2}}{2 \cdot a_{t} \cdot l_{t}}\right)
$$

The actual angle displacement of the swing rod is given by Equation (5).

$$
\varepsilon=\Delta \varepsilon+\varepsilon_{\min }
$$

When the step size is smaller, an accurate cam theoretical contour curve can be obtained by Equation (6). The actual 
contour curve of the cam is obtained by Equation (7).

$$
\begin{gathered}
\left\{\begin{array}{l}
x_{t}=a_{t} \cdot \sin (\delta)-l_{t} \sin G(\delta)+\varepsilon_{\min } \\
y_{t}=a_{t} \cdot \cos (\delta)-l_{t} \cos G(\delta)+\varepsilon_{\min }
\end{array}\right. \\
\left\{\begin{array}{l}
x_{t 1}=x_{t}+r r \cdot \frac{d y_{t}}{\sqrt{d y_{t}^{2}+d x_{t}^{2}}} \\
y_{t 1}=y_{t}-r r \cdot \frac{d x_{t}}{\sqrt{d y_{t}^{2}+d x_{t}^{2}}}
\end{array}\right.
\end{gathered}
$$

The length $b$ is determined by the structure of the angle magnification mechanism in the double planet carrier planetary gear mechanism, mm; The length $d$ is equal to $l$ minus $b, \mathrm{~mm}$. Obtain the actual angle displacement of the transplanting arm is $\gamma=k G(\delta)$.

The trajectories of the point $p^{\prime}$ and the trajectories of the point $m$ are obtained by Equations (8) and (9).

$$
\begin{gathered}
\left\{\begin{array}{l}
x_{p^{\prime}}=x_{0}+r \cdot \cos \left(\varphi_{1}^{\prime}\right)+d \cdot \cos \left(\varphi_{2}^{\prime}\right) \\
y_{p^{\prime}}=y_{0}+r \cdot \sin \left(\varphi_{1}^{\prime}\right)+d \cdot \sin \left(\varphi_{2}^{\prime}\right)
\end{array}\right. \\
\left\{\begin{array}{l}
x_{m}=x_{p^{\prime}}+b \cdot \cos \left(\varphi_{2}^{\prime}+\gamma\right) \\
y_{m}=y_{p^{\prime}}+b \cdot \sin \left(\varphi_{2}^{\prime}+\gamma\right)
\end{array}\right.
\end{gathered}
$$

\section{Optimal design of the transplanting mechanism}

\subsection{Working requirements of flower transplanting mechanism}

The flower transplanting mechanism must complete four operations of picking seedling, seedling delivery, planting seedling and return trip within a cycle. The cooperative work of the flower transplanting mechanism, seedling box and conveyor belt is shown in Figure 6. The first planet carrier of the mechanism rotates counterclockwise.

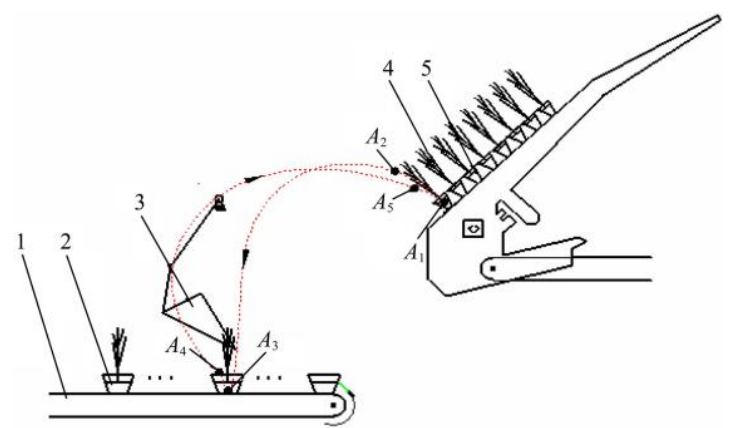

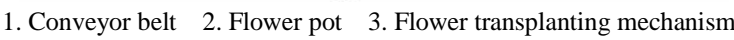

4. Flower plug seedling 5. Seedling box

Figure 6 Schematic diagram of the operation of the flower transplanting mechanism

1) $A_{1}$ to $A_{2}$ : This trajectory segment is the stage of picking seedlings out of seedling box. The transplanting needle clamps the flower plug seedling at $A_{1}$. This trajectory segment needs to ensure that the transplanting needle can take out the plug seedlings along the vertical direction of the seedling box.

2) $A_{2}$ to $A_{3}$ : This trajectory segment is the stage of delivering seedlings and planting seedling into flower pots. The transplanting needles clamp the flower plug seedling all the time in this trajectory segment. The transplanting needles retracted at $A_{3}$, and the flower plug seedlings are detached from the transplanting needles. In order to ensure that flower plug seedling in the vertical state when planted in a flower pot, the transplanting needles should be vertical to the flower pot at $A_{3}$.

3) $A_{3}$ to $A_{4}$ : This trajectory segment is the stage of the retraction of the transplanting needle. The transplanting needles should retract quickly to prevent them from pushing over the empty flower pots that are transported on the conveyor belt.

4) $A_{4}$ to $A_{5}$ : This is the return trajectory segment. The transplanting needles are in the retracted state, and there is no special requirement in this part.

5) $A_{5}$ to $A_{1}$ : This trajectory segment is the stage of the seedling picking state. The transplanting needles gradually extend in the transplanting arm and insert from under the seedling box.

\subsection{Optimal design of the mechanism}

Based on the trajectory characteristics of the single planetary carrier mechanism, it can be known that the terminal of the linkage of the single planetary carrier mechanism with second-order non-circular gear must pass the farthest point and the shortest point of the trajectory twice. This feature can be used to design a large displacement transplanting mechanism.

The horizontal angle between the seedling box and the ground is $60^{\circ}$, based on the previous analysis of work requirements, the angle between the transplanting arm and the flower plug seedling is set to $25^{\circ}$, that is, the included angle of the mark line is $25^{\circ}$. In order to have a large displacement in both the $x$-direction and the $y$-direction, the rotate angle of the first planet carrier between the two farthest trajectory points should be within $80^{\circ}-100^{\circ}$, the angle is set to $95^{\circ}$ in this design.

The coordinate of the rotation center of the mechanism is set to $(0,0)$, the coordinate of $P_{1}$ is $(327.8,-28.7)$, and the angle of $P_{1}$ is $175^{\circ}$. The angle between $P_{1} O$ and $P_{3} O$ is $95^{\circ}$, the angle of $P_{3}$ is $80^{\circ}$, and the angle of the mark line of $P_{1}$ and $P_{3}$ is determined as shown in Figure 7 a.
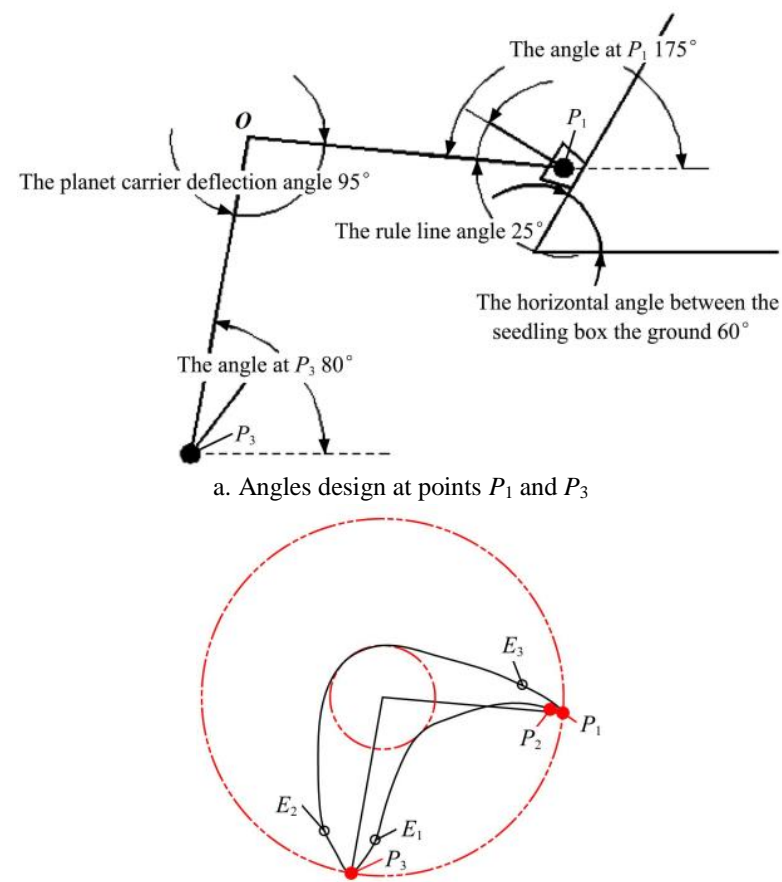

b. Preliminary planned trajectory

Figure 7 Trajectory planning of the single planetary carrier mechanism

Designing the preliminary planned trajectory in CAD as shown in Figure $7 \mathrm{~b}$, the trajectory is formed clockwise. On the trajectory, select another precise attitude point $\mathrm{P}_{2}$ near the point $P_{1}$, and obtain the coordinates of this point. Since this point is close to $P_{1}$, the trajectory of $P_{1}$ to $P_{2}$ is still located inside the seedling box. Considering that the angle of the transplanting needles cannot be kept constant, the attitude angle of $P_{2}$ is set to $164^{\circ}$. Three trajectory shape control points $E_{1}, E_{2}$, and $E_{3}$ are specified on the trajectory. The transplanting needle is completed entering the seedling box and picking seedling work in section $E_{3} P_{1} P_{2}$, seedlings are planted in section $E_{1} P_{3} E_{2}$. 
The relevant position and angle of $P_{2}, E_{1}, E_{2}$, and $E_{3}$ are shown in Table 1.

Table 1 Three precise attitude points and trajectory control points of the single planetary carrier mechanism

\begin{tabular}{ccc}
\hline Control point & Position $\left(x_{i}, y_{i}\right) / \mathrm{mm}$ & Angle $\theta /\left(^{\circ}\right)$ \\
\hline$P_{1}$ & $(327.8,-28.7)$ & 175 \\
$P_{2}$ & $(314.6,-25)$ & 164 \\
$P_{3}$ & $(-57,-323.8)$ & 80 \\
$E_{1}$ & $(-12.8,-263.5)$ & $/$ \\
$E_{2}$ & $(-105.3,-246.8)$ & $/$ \\
$E_{3}$ & $(254,21.9)$ & $/$ \\
\hline
\end{tabular}

The length range of the first planetary carrier is $100-140 \mathrm{~mm}$ and the length of the linkage is $145-220 \mathrm{~mm}$, the range of the $x$-axis of the rotation center of the planetary carrier is -50 to $50 \mathrm{~mm}$ in paper. Hinge points' solution domain of the open chain two-linkage group is shown in Figure 8. (both are solution domain diagrams with removing motion defects)

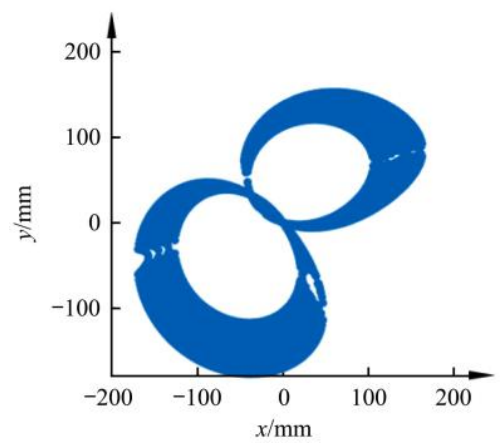

a. Solution domain of the fixed hinge point

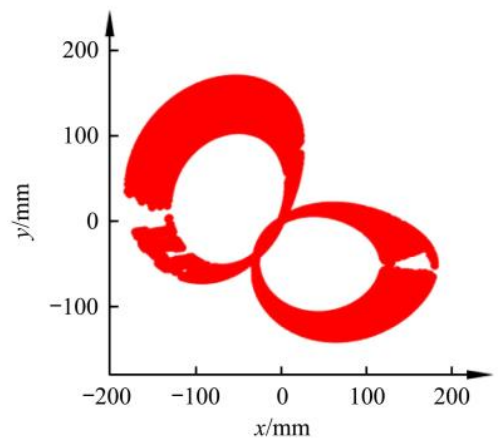

b. Solution domain of the moving hinge point

Figure 8 Diagram of the solution of the rod group

Considering the structure of the double planet carrier planetary gear flower potting transplanting mechanism, the length ratio of the linkage and the first planet carrier is set within the range of 1.35-2. According to the conditions of non-circular gear pitch curve without depression and ring buckle. A suitable set of hinge solution is determined in the domain (Table 2). Then the transmission ratio of non-circular gear train based on two linkages' relative angle relationship and gear profile of the second-order non-circular gear pitch can be obtained. Thus, the design of single planetary train mechanism is completed, and its trajectory is shown in Figure 9.

The basic trajectory shape shown in Figure 9 meets the transplanting requirements, but the seedling is $70^{\circ}$ away from the vertical orientation and the vertical planting target cannot be achieved without damaging the gear smoothing design, so a double planetary carrier and cam gear are introduced to resolve this dilemma. Multiple points on trajectory are set and associated with the motion of the cam, it is determined that $F_{3}-F_{7}$ is the trajectory near the point of picking seedling, $F_{0}-F_{2}$ is the trajectory near the point of planting seedling. According to the trajectory design requirements of the flower transplanting, the trajectory of the terminal of the transplanting needles should form a small ring during the picking seedling segment. Therefore, from $F_{3}$ to $F_{4}$, the angle of the swing rod should be 0 . In $F_{4}-F_{0}$, multiple points are selected so that the trajectory of $F_{4}$ to $F_{0}$ segment is slightly higher than those of $F_{3}$ to $F_{4}$ segment in the vertical direction.

Table 2 Parameter of the single planet carrier linkage group

\begin{tabular}{lc}
\hline \multicolumn{1}{c}{ Parameter } & Values \\
\hline The fixed hinge point $O / \mathrm{mm}$ & $(27,-48.1)$ \\
The moving hinge point $Q_{c 1} / \mathrm{mm}$ & $(-38.36,-148.74)$ \\
The length of the first planet carrier $r / \mathrm{mm}$ & 120 \\
The length of the linkage $l / \mathrm{mm}$ & 181.65 \\
Initial angle of the first planet carrier $/\left(^{\circ}\right)$ & 236.99 \\
Initial angle of the linkage $/\left(^{\circ}\right)$ & 26.99 \\
\hline
\end{tabular}

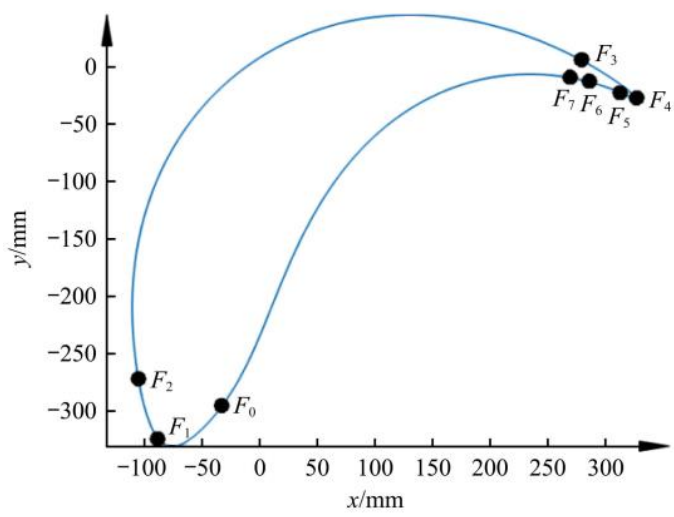

Figure 9 Basic trajectory of single planetary train mechanism

Segment $F_{4}$ to $F_{0}$ corresponds to the lift section of the cam that drives the transplant arm, segment $F_{0}$ to $F_{2}$ corresponds to the distal stop section, segment $F_{2}$ to $F_{3}$ corresponds to the return section, and segment $F_{3}$ to $F_{4}$ corresponds to the proximity stop section. The maximum rotate angle of the swing rod is set as $35^{\circ}$, combined with the linkage angle relative to the first planetary carrier, the cam gear parameters at each specified point are listed in Table 3.

Table 3 Design parameters of cam gear

\begin{tabular}{cccc}
\hline $\begin{array}{c}\text { Angle control } \\
\text { points }\end{array}$ & $\begin{array}{c}\text { The cam } \\
\text { rotation } \\
\text { angles } /\left(^{\circ}\right)\end{array}$ & $\begin{array}{c}\text { The cam rotation } \\
\text { angles entered in the } \\
\text { program } /\left(^{\circ}\right)\end{array}$ & $\begin{array}{c}\text { The rotation angles } \\
\text { of the swing } \\
\text { lever/ } /\left(^{\circ}\right)\end{array}$ \\
\hline$F_{0}$ & 15 & 15 & 35 \\
$F_{1}$ & 28.4 & 28.4 & 35 \\
$F_{2}$ & 65 & 65 & 35 \\
$F_{3}$ & 160 & 160 & 0 \\
$F_{4}$ & 180 & -180 & 0 \\
$F_{5}$ & 199 & -161 & 0.5 \\
$F_{6}$ & 205 & -155 & 1.5 \\
$F_{7}$ & 213 & -147 & 3.5 \\
\hline
\end{tabular}

The magnifies factor $k$ of the circle gear magnification mechanism inside the second planetary carrier is 2 , the center distance of the cam gear is $40 \mathrm{~mm}$, the teeth in a pair of circle gears are 13 and 26, and the gear center distance is $29.25 \mathrm{~mm}$, therefore the actual length of the second planet carrier $b$ is $69.25 \mathrm{~mm}$, the length of the transplanting arm $d$ is $112.4 \mathrm{~mm}$.

The comparison of the two trajectories is shown in Figure 10. The improved trajectory formed a ring buckle at the picking seedling segment. The existence of ring buckle will allow the needle to enter from the bottom of the seedling when the 
mechanism takes seedlings. It can reduce the contact with the seedlings, thereby reducing the damage to the seedlings and increasing the success rate of transplanting. Especially, the transplanting arm of the double planet carrier planetary gear mechanism can theoretically plant seedlings vertically at the lowest point of the improved trajectory. When the solid line trajectory is at the lowest point of transplanting, there is an angle deviation between the second rack and the third rack, and the transplanting displacement will be slightly smaller than the theoretical trajectory. The additional parameters of the mechanism due to structural changes are listed in Table 4.

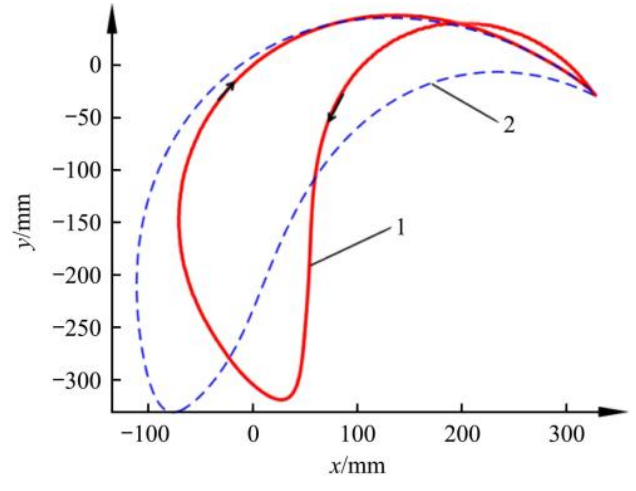

1. Improved trajectory 2 . Basic trajectory

Figure 10 Comparison of the two trajectories

Table 4 Additional parameters of the double planet carrier planetary gear flower transplanting mechanism

\begin{tabular}{lc}
\hline \multicolumn{1}{c}{ Additional parameters } & Values \\
\hline Actual end point of the second planet carrier $\left(x_{p^{\prime}}, y_{p^{\prime}}\right) / \mathrm{mm}$ & $(-49.19,-217.14)$ \\
Terminal of the transplanting arm $\left(x_{y m}, y_{y m}\right) / \mathrm{mm}$ & $(48.35,-272.99)$ \\
The length of the second planet carrier $d / \mathrm{mm}$ & 69.25 \\
The length of the transplanting arm $b / \mathrm{mm}$ & 112.4 \\
Initial angle of the transplanting $\operatorname{arm} /\left(^{\circ}\right)$ & 330.2 \\
\hline
\end{tabular}

\section{Design and experiment of the transplanting} mechanism

5.1 Prototype of the mechanism and its kinematic verification

Figure 11 is $3 \mathrm{D}$ model of a double planetary gear flower transplanting mechanism. The first planetary carrier has a non-circular gear train containing a second-order non-circular gear.

Based on the $3 \mathrm{~d}$ model, the structure rationality of the mechanism is checked, and the correctness of the mechanism design is verified by the virtual simulation of the mechanism, then the test prototype of the mechanism was produced.

Idling experiment of the mechanism is carried out, and the idling experiment is photographed by high-speed camera. The motion video is imported into the photographic analysis software to obtain the trajectory shown in Figure 12.

The actual trajectory is similar to the improved trajectory of Figure 10, but there is still a subtle difference. The reason for this difference is that the transplanting needles will retract and extend in the actual experiment, and the calculation of the theoretical trajectory is the trajectory formed when the transplanting needles are stretched out. The trajectory of idling experiment at the stage of planting seedling is slightly sharper than the improved trajectory. In experiment, the reset spring connecting the transplanting arm with the second planet carrier is not fully tightened, which leads to the angle displacement between the transplanting arm and the second planet carrier is deviated from the theory. When planting seedlings and picking seedlings, the azimuth angle of the mechanism was still the same as the theoretical design and did not affect the actual work effect.

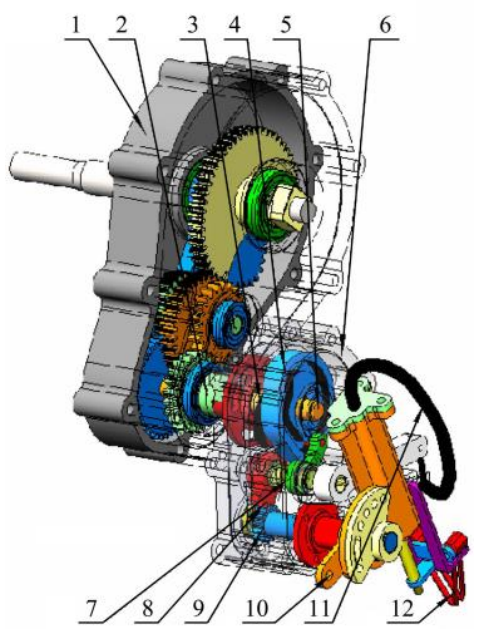

1. First planet carrier 2. Second medium gear 3. Cam 4. Bracing wire driven cam 5. planet carrier shaft 6. Second planet carrier 7. Wire rod 8. Swing rod 9. Transplanting arm gear shaft 10 . Transplanting arm 11. Bracing wire 12. Transplanting needle

Figure 11 3D model of flower transplanting mechanism

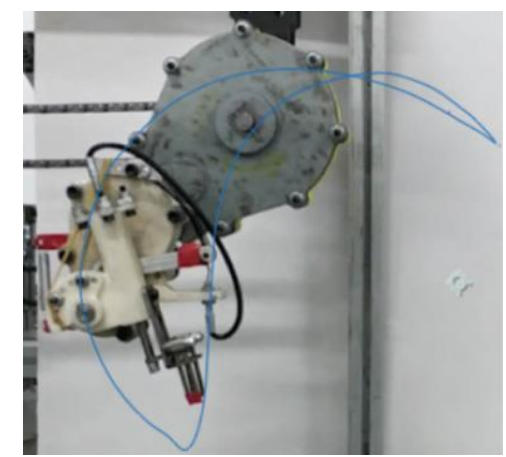

Figure 12 Actual trajectory of idling experiment

\subsection{Flower seedling transplanting experiment}

A flower transplanting experiment was carried out with collard plug seedling. The seedling age was $45 \mathrm{~d}$, the diameter of the outer diameter of the flower pot was $140 \mathrm{~mm}$, and the specification of the plug seedling box was 16 rows and 8 columns, with a total of 128 acupoints. The efficiency of transplanting was 40 seedlings $/ \mathrm{min}$. A total of 4 groups of transplanting experiments were carried out, and the experiment results are shown in Table 5.

Table 5 Results of flower transplanting experiment

\begin{tabular}{cccc}
\hline $\begin{array}{c}\text { Experiment } \\
\text { No. }\end{array}$ & $\begin{array}{c}\text { Numbers of seedling } \\
\text { taking }\end{array}$ & $\begin{array}{c}\text { Numbers of } \\
\text { successful planting }\end{array}$ & Successful rate \\
\hline 1 & 126 & 120 & $95.24 \%$ \\
2 & 120 & 112 & $93.33 \%$ \\
3 & 122 & 116 & $95.08 \%$ \\
4 & 118 & 111 & $94.07 \%$ \\
\hline
\end{tabular}

The average success rate of seedlings picking is $94.43 \%$ and the success rate of the four experiments are $95.24 \%, 93.33 \%$, $95.08 \%$ and $94.07 \%$, respectively. A total of 25 seedlings failed to be planted, 9 times for the oversized leaves were taken out by neighboring seedlings, 7 times were failed to picking out plug seedling, 5 times for the seedling separated from the substrate, and 4 times for the loss of plug seedlings during seedling transportation. In the experiment, it was found that there is slight contact between the black wire and the seedling when some seedlings were huge, but it has little effect on the success rate of the experiment. The result indicated that seedling quality, seedling leaf size control, 
appropriate increase of seedling picking force at picking point could further increase the performance of the mechanism.

The mechanism attitude when the seedling is picked and planted is shown in Figure 13, the posture of the seedling needle in these two positions reached the design target. According to the statistics of the main stem angle of seedling planted in a flowerpot, it was found that $90.07 \%$ of the seedlings' angle is in the range of $80^{\circ}-100^{\circ}$, which show that the mechanism has high planting uprightness.

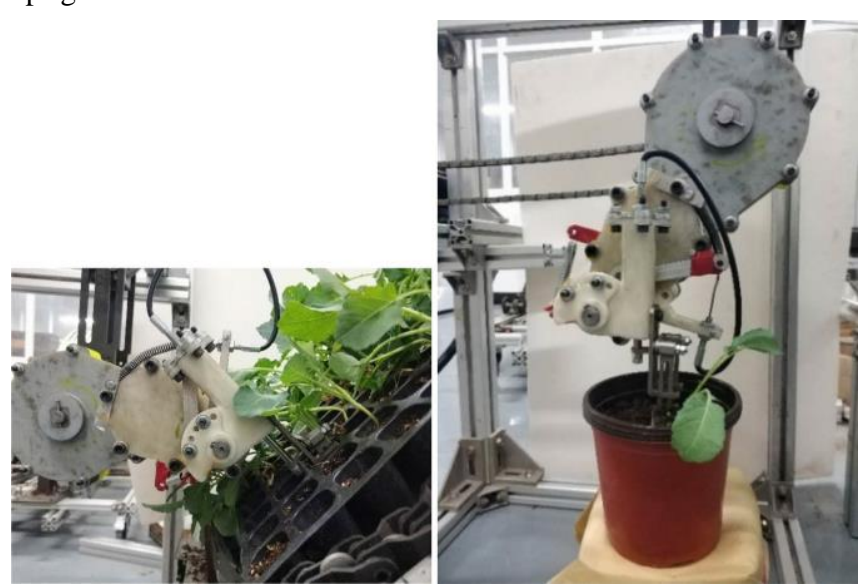

Figure 13 Seedling-picking and planting attitude of double planet carrier planetary gear mechanism

\section{Conclusions}

In view of the shortcomings of the existing controlled and single planetary carrier transplanting mechanism, a double planet carrier planetary gear mechanism combining a planetary carrier and a cam gear was proposed. The second planet carrier of the mechanism performs variable-speed rotation relative to the first planet carrier, and the transplanting arm performs variable-speed swing relative to the second planet carrier.

A mathematical model of a double planet carrier planetary gear mechanism was established. Combined with the trajectory requirements of flower potting operations, a set of optimized parameter solutions to this type of mechanism was obtained. The length of the planet carrier is $120.00 \mathrm{~mm}$, the second planet carrier is $69.25 \mathrm{~mm}$, and the transplanting arm is $112.40 \mathrm{~mm}$. From the theoretical trajectory of the mechanism, the amount of transplanting displacement reached $298.05 \mathrm{~mm}$.

The double planet carrier planetary gear mechanism was designed and developed. The actual transplanting trajectory and theoretical design trajectory were compared and analyzed. The correctness of the design method of the double planet carrier planetary gear train was verified. The success rate of the double planet carrier planetary gear mechanism reached $94.43 \%$. The plug seedlings planted in flowerpots have high uprightness.

\section{Acknowledgements}

This work was financially supported by the National Key Research and Development Program of China (Grant No. 2017YFD0700800); the National Natural Science Foundation of China (Grant No. 32071909, No. 51775512); Basic public welfare research projects of Zhejiang Province (Grant No. LGN19E050002, No. LGN20E050006) and Fundamental Research Funds of Zhejiang Sci-Tech University (Grant No. 2020Q013).

\section{[References]}

[1] Zhang Y Y, Yu L, Liu J N, Hu J X, Hua J Z, Yao L Y, et al. Status quo and enhancement of competitiveness of cut flower industry in China. Agricultural Science \& Technology, 2017; 18(2): 368-371. (in Chinese)

[2] Feng Q C, Wang X, Jiang K, Zhou J J, Zhang R, Ma W. Design and test of key parts on automatic transplanter for flower seedling. Transactions of the CSAE, 2013; 29(6): 21-27. (in Chinese)

[3] Hao C Y, Wang L J. Based on PLC flower bowl seedlings transplanting machine automatic transmission device. Journal of Agricultural Mechanization Research. 2018; 40(6): 220-223. (in Chinese)

[4] Zhao X, Zhang X S, Wu Q P, Dai L, Chen J N. Research and experiment of a novel flower transplanting device using hybrid-driven mechanism. Int $\mathrm{J}$ Agric \& Biol Eng, 2020; 13(2): 92-100.

[5] Ting K C, Giacomell G A, Shen S J, Kabala W P. Robot workcell for transplanting of seedlings part II end-effector development. Transactions of the ASABE, 1990; 33(3): 1013-1018.

[6] Dang Y G, Jin X, Li H J, Wang J, Lu Y B, Ding B W, et al. Design of single-degree-of-freedom four-bar seedling-taking and throwing manipulator. Transactions of the CSAE, 2019; 35(14): 39-47. (in Chinese)

[7] Choi W C, Kim D C, Ryu I H. Development of a seedling pick-up device for vegetable transplanters. Transactions of the ASAE, 2002; 45(1): 13-19.

[8] Xin L, Lyu Z J, Wang W Q. Optimal design and development of a double-crank potted rice seedling transplanting mechanism. Transactions of the ASAE, 2017; 60(1): 31-40.

[9] Zhou M F, Xu J J, Tong J H, Y G H, Zhao X, Xie J. Design and experiment of integrated automatic transplanting mechanism for taking and planting of flower plug seedlings. Transactions of the CSAE, 2018; 34(20): 44-51. (in Chinese)

[10] Ye B L, Li L, Yu G H, Liu A, Zhao Y. Dynamics analysis and test of rotary pick-up mechanism for vegetable pot-seedling. Transactions of the CSAM, 2014; 45(6): 70-78. (in Chinese)

[11] Yu G H, Jin Y, Chang S S, Ye B L, Gu J B, Zhao X. Design and test of clipping-plug type transplanting mechanism of rice plug-seedling. Transactions of the CSAM, 2019; 50(7): 100-108. (in Chinese)

[12] Yu G H, Liao Z P, Xu L H, Zhao P, Wu C Y. Optimization design and test of large spacing planetary gear train for vegetable pot-seedling planting mechanism. Transactions of the CSAM, 2015; 46(7): 38-44. (in Chinese)

[13] Sun L, Xu Y D, Huang H M, Wang Z F, Zhang G F, Wu C Y. Solution and analysis of transplanting mechanism with planetary gear train based on convexity of pitch curve. Transactions of the CSAM, 2018; 49(12): 83-92. (in Chinese)

[14] Xue X L, Wang L, Xu C L, Zhou M L, Zhao Y. Optimal design and experiment of longitudinal feeding-seedling device of potted seedling transplanter for upland field. Transactions of the CSAM, 2020; 51(2): 76-84. (in Chinese)

[15] Zhao X, Li K W, Cui H Y, Chen J N, Ye B L, Dai L. Design method and applied research of a planetary gear system mechanism based on solution region synthesis. International Agricultural Engineering Journal, 2019; 28(2): 100-109.

[16] Zhao X, Ye J, Chu M Y, Dai L, Chen J N. Automatic scallion seedling feeding mechanism with an asymmetrical high-order transmission gear train. Chinese Journal of Mechanical Engineering, 2020; 33: 10. doi: 10.1186/s10033-020-0432-9.

[17] Zhao Y, Zhang W X, Xin L, Xie J T, Xue X L, Shan Y Y. Design and experiment of extensible potted tomatoes seedling transplanting mechanism. Transactions of the CSAM, 2019; 50(1): 112-119. (in Chinese)

[18] Sun L, Shen J H, Zhou Y Z, Ye Z Z, Yu G H, Wu C Y. Design of non-circular gear linkage combination driving type vegetable pot seedling transplanting mechanism. Transactions of the CSAE, 2019; 35(10): 26-33. (in Chinese)

[19] Choi W C, Kim D C, Ryu I H, et al. Development of seedling pick-up device for vegetable transplants. Transactions of the ASAE, 2002; 45(1): 13-19.

[20] Yang T, Han J Y. Solution region synthesis method of planar one-DoF six-bar linkages for specifying 4-positions of a 3R open chain. Journal of Mechanical Science \& Technology, 2016; 30(7): 3069-3077. 